\title{
Stress-Wave Induced Fracture of Unidirectional Composites: An Experimental Study using Digital Image Correlation method
}

\author{
Dongyeon Lee ${ }^{1}$ and Hareesh V. Tippur ${ }^{2}$ \\ Auburn University, Auburn, AL 36849, USA
}

Fracture behavior of unidirectional graphite/epoxy composite materials is investigated. Single-edge notched coupons are studied under geometrically symmetric impact loading. The notch orientation parallel to or at an angle relative to the fiber orientation is considered to produce mode-I as well as mixed-mode fracture. Stress-wave induced crack initiation and rapid crack growth events are studied using the digital correlation technique and high-speed photography. Surface deformations histories in the crack-tip vicinity are obtained by analyzing speckle image recordings. Measured deformation fields are used to extract mixedmode fracture parameters and examine the effect of fiber orientation on crack initiation and growth behaviors. The maximum crack speed observed is the highest for mode-I dominant conditions and decreases with increasing fiber orientation angle. With increasing fiber orientation angle, crack takes longer to attain the maximum speed upon initiation. The crack initiation toughness values decrease with increasing degree-of-anisotropy.

\section{Introduction}

In view of the vast range of strain-rates fiber reinforced plastics encounter during service life, it is important to study the fracture behavior under elevated rates of loading. The past studies have primarily focused on quasi-static or mode-I fracture behavior of unidirectional composite laminates containing through-thethickness notches. Very few studies have been carried out for investigating the mode-II or mixed-mode conditions. Some of the previous works have used optical methods such as moiré interferometry [1], lateral shearing interferometry [2], and laser-speckle interferometry [3] to study the problem. Since full-field measurements can be made in real-time using these techniques, transient events can be investigated. However, surface preparation required for implementing these techniques is often quite challenging. Accordingly the present work is an attempt to investigate mixed-mode dynamic fracture behavior of multilayered unidirectional composites using the method of digital image correlation coupled with high-speed photography. This experimental method offers the advantage of relative simplicity in terms of surface preparation of specimens and recent developments in digital imaging technology can be further exploited beneficially to study this challenging problem.

\footnotetext{
${ }^{1}$ Post-doctoral Fellow, Department of Mechanical Engineering, 270 Ross Hall, Auburn University, AL 36849, USA

${ }^{2}$ Professor of Mechanical Engineering, 270 Ross Hall, Auburn University, AL 36849, USA
} 
In the current work, dynamic crack growth in composite laminates was produced in edge-notched rectangular samples with different fiber orientations by impact loading. Fast fracture at crack velocities of several hundred meters per second was captured using a digital high-speed camera. The method of digital image correlation (DIC) [4, 5] was employed in conjunction with high-speed photography to examine full-field surface-displacements in the vicinity of a rapidly growing crack and subsequently extract fracture parameters. It is worth noting that, due to the unavailability of ultra high-speed digital cameras capable of digitizing image intensities at relatively high resolution until quite recently, DIC has not been extensively used to study stress-wave induced failures in general and dynamic fracture mechanics of fiber reinforced composites in particular. Accordingly, this work introduces novelty in terms of extending DIC to study dynamic fracture mechanics of fiber reinforced composites using digital high-speed photography.

\section{Displacement Fields Under Mixed Loading}

Let a planar unidirectional composite with a crack be subjected to mixed mode (mode-I and mode-II) static loading. Then the in-plane displacement components in the vicinity of crack tip of the material are given by [6]:

$$
\begin{aligned}
& u_{x}=K_{I} \sqrt{\frac{2 r}{\pi}} \operatorname{Re}\left[\frac{1}{\mu_{2}-\mu_{1}}\left(p_{1} \mu_{2} z_{1}-p_{2} \mu_{1} z_{2}\right)\right]+K_{I I} \sqrt{\frac{2 r}{\pi}} \operatorname{Re}\left[\frac{1}{\mu_{2}-\mu_{1}}\left(p_{1} z_{1}-p_{2} z_{2}\right)\right]+\cdots \\
& u_{y}=K_{I} \sqrt{\frac{2 r}{\pi}} \operatorname{Re}\left[\frac{1}{\mu_{2}-\mu_{1}}\left(q_{1} \mu_{2} z_{1}-q_{2} \mu_{1} z_{2}\right)\right]+K_{I I} \sqrt{\frac{2 r}{\pi}} \operatorname{Re}\left[\frac{1}{\mu_{2}-\mu_{1}}\left(q_{1} z_{1}-q_{2} z_{2}\right)\right]+\cdots
\end{aligned}
$$

where $u_{x}$ represents the sliding displacement, $u_{y}$ the opening displacement, and quantities $p_{j}, q_{j}$, and $z_{j}(j=1,2)$ are defined as:

$$
\begin{aligned}
& p_{j}=\mu_{j}^{2} s_{11}+s_{12}-\mu_{j} s_{16} \\
& q_{j}=\mu_{j} s_{12}+\frac{s_{22}}{\mu_{j}}-s_{26} \\
& z_{j}=\sqrt{\cos \theta+\mu_{j} \sin \theta} \\
& s_{i j}(i, j=1, \ldots, 6) \text { are the elements of compliance matrix. }
\end{aligned}
$$

Subscripts 1 and 2 denote the fiber direction and in-plane cross-fiber direction, respectively. The coordinates $x$ and $y$ are the two principal directions of a composite sample as illustrated in Fig. 1. Here $r$ is the radial distance from the crack tip, $\theta$ the angle with respect to the $x$-axis, and $\beta$ the fiber orientation angle. 


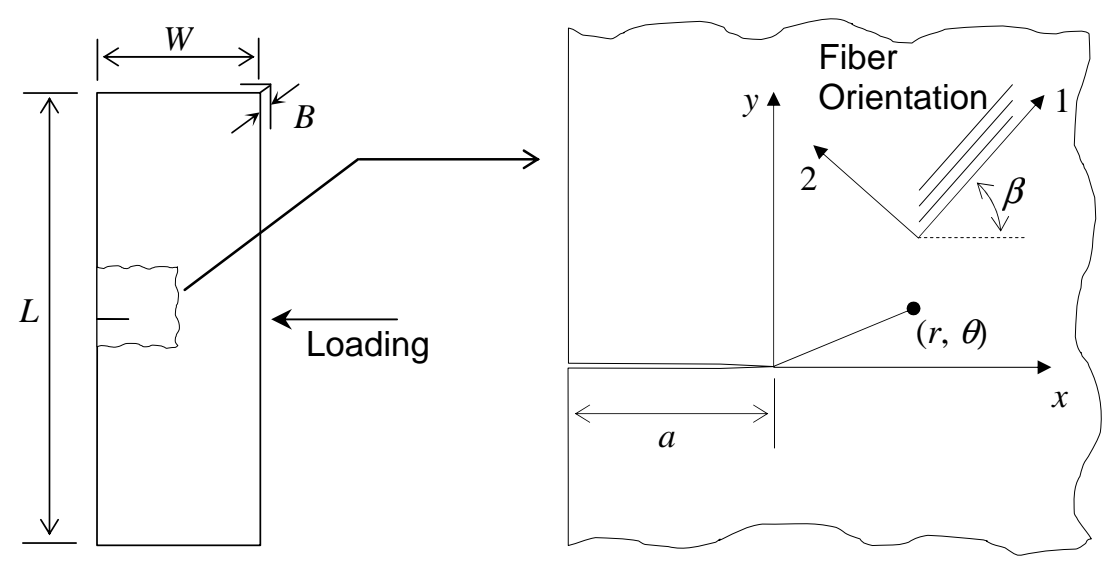

Figure 1. Illustration of crack-tip neighborhood $(L=200 \mathrm{~mm}, W=50 \mathrm{~mm}, a=$ $0.2 W, B=6.2 \mathrm{~mm})$.

Quantities $\mu_{j}(j=1,2)$ are the two roots of the characteristic equation,

$s_{11} \mu^{4}-2 s_{16} \mu^{3}+\left(2 s_{12}+s_{66}\right) \mu^{2}-2 s_{26} \mu+s_{22}=0$

with positive imaginary part (the other two are conjugates of these roots). For general anisotropic materials and orthotropic materials, roots are complex numbers and pure imaginary numbers, respectively. It should be noted that, at the moment, similar expressions for crack tip displacements for the case of a dynamically growing crack in a unidirectional composite sheet is only limited to mode-I conditions.

\section{Optical Method}

DIC method involves recording gray scales of random pattern in the undeformed and deformed states. The relative change in the position and distortion of subimages of the image are evaluated to find displacements and displacement gradients. In the current work, a three-step DIC approach $[4,5]$ implemented in a MATLAB $^{\mathrm{TM}}$ environment was used to estimate in-plane displacements. First, an initial estimation of displacements was made using a 2D cross-correlation between two corresponding sub-images of undeformed and deformed states using fast Fourier transforms [7]. The peak of the correlation function was detected to a subpixel accuracy using bicubic interpolation. The process was repeated for the entire image to estimate in-plane displacements, $u_{x}$ and $u_{y}$, over an array of grid points. At this stage, however, displacement field contains significant random noise. In the second step, the previously obtained displacements were refined by minimizing a 2D correlation coefficient [8] iteratively using displacements from the first step as initial guesses. In this step, a significant amount of noise present 
in the earlier displacement estimation was removed. Finally, smoothing algorithms based on regularized restoration filter with second order fit was applied to the displacement fields separately. The smoothing process employed in the present work allows discontinuity of displacements across the crack faces. Details on the smoothing process are reported in Ref. [5].

The experimental set-up consisted of an instrumented drop tower for delivering low-velocity impact and a high-speed digital camera for capturing images. The impact force and support reaction histories during impact were recorded. Two high-intensity flash lamps, triggered by the camera, were used to illuminate the gray scale patterns. The recording devise was a rotating mirror-type high-speed camera with multiple CCD arrays. The camera is capable of capturing images up to $2 \times 10^{6}$ frames per second at a resolution of $1,000 \times 1,000$ pixels per image. There are 32 independent CCD arrays positioned circumferentially around a fivefacet rotating mirror, which reflects and sweeps light over the sensors. Each sensor is illuminated by a separate optical relay consisting of separate lenses and partial mirrors.

Prior to impacting the specimen, a set of 32 images of the region-of-interest was photographed at a desired framing rate (250,000 frames per second in the current work). After aligning the optics, the rotating mirror was brought to the desired speed. The camera and flash lamps were triggered externally by the operator. This produceed a set of 32 images of the undeformed specimen surface. While maintaining all camera settings unchanged, a second set of 32 images is photographed as the specimen was subjected to impact loading (impact speed $=$ $4.8 \mathrm{~m} / \mathrm{sec}$ ): i.e., another set of 32 images of the deformed specimen surface. Thus, for every image in the deformed image set, there was a corresponding image in the undeformed set.

Fracture specimens were prepared from sheets made of hot-compression molded T800/3900-2 prepreg tapes. A total of 35 plies were stacked together, resulting in $6.2-\mathrm{mm}$ thickness, with fibers aligned in one direction. Panels were cut into 200 $\mathrm{mm} \times 50 \mathrm{~mm}$ rectangular coupons with four different fiber orientations, $0^{\circ}, 15^{\circ}$, $30^{\circ}$, and $45^{\circ}$, relative to the width of specimens. The material properties are: $E_{1}=$ $171.6 \mathrm{GPa}, E_{2}=8.25 \mathrm{GPa}, G_{12}=6.21 \mathrm{GPa}$, and $v_{12}=0.344$, in which subscripts 1 and 2 represent the directions parallel and (in-plane) perpendicular to the fiber orientation, respectively (see Fig. 1). Each specimen was then pre-notched by a $0.3-\mathrm{mm}$ thick diamond wafer blade followed by a razor blade for further sharpening. The initial pre-notch of length $10 \mathrm{~mm}$ was made along the line-ofsymmetry at the sample edge and oriented along the impacting direction. Prenotched samples were then sprayed with black and white paint to obtain a random speckle pattern. 


\section{Results}

A few representative speckle images are shown in Fig. 2. Dynamic crack growth along the fibers is clearly visible from the lower image and the crack tip is identified by an arrow. From the displacement matrices for each time step, the inplane displacement contour plots (contour intervals $2 \mu \mathrm{m}$ ) were obtained. The corresponding $u_{y}$-field and $u_{x}$-field for the images are also shown in Fig. 2. The discontinuity in displacements at the crack tip is evident across the crack faces in each image. The accuracy of displacement measurements is $\sim 1.8 \mu \mathrm{m}$ as detailed in Ref. [5]. These displacement values are used next along with Eq. (1) to compute stress intensity factors.
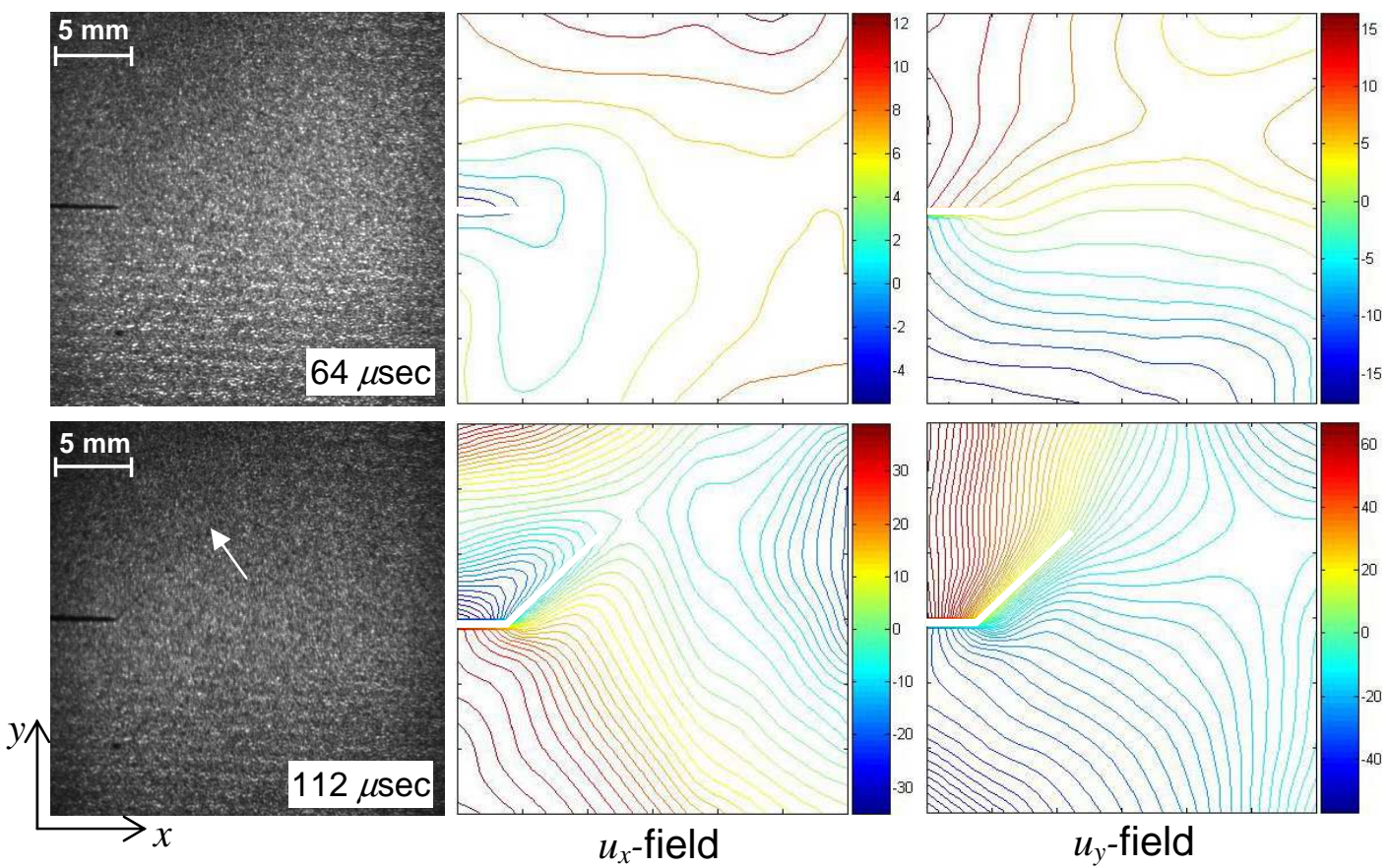

Figure 2. Representative speckle images for $\beta=45^{\circ}$ case $(30 \mathrm{~mm} \times 30 \mathrm{~mm})$ with full-field $u_{x}$ and $u_{y}$ displacement contours (crack initiation takes place at $t \sim 84 \mu \mathrm{s}$ ). Moving crack tip is indicated by an arrow and the dark line is the initial pre-notch in photographed images. Color-bar indicates displacement in micrometers.

Crack-tip velocity histories were computed from the crack length history data for each case using smoothed cubic Bezier curve (smoothing parameter of 0.5). The crack-tip velocities were determined from the fitted curves. The maximum velocity in these cases is in the range of $460-560 \mathrm{~m} / \mathrm{sec}$, with the highest velocity for $\beta=0^{\circ}$. The peak of velocity occurs earlier for $\beta=0^{\circ}$, and progressively later for $15^{\circ}, 30^{\circ}$, and $45^{\circ}$. This can be explained in terms of increasing shear deformations as fiber orientation increases. For a pre-notch that is not along the fiber orientation, a significant portion of the accumulated energy at the crack tip is 
consumed by the crack initiation event due to the fibers bridging across the crack flanks. In view of this, even after crack initiation, it takes relatively longer time to reach the maximum velocity for higher $\beta$ values. Furthermore, following crack initiation, the crack-tip velocity in each case continues to increase monotonically for longer durations with increasing fiber orientation: the shortest duration is for the $0^{\circ}$ case and the longest for the $45^{\circ}$ case. Once the crack has attained the peak velocity, a decreasing trend exists due to increasing shear component as well as the compression dominated impact region.

\section{Discussion}

Stress intensity factors were evaluated from measured displacement fields. Data was collected in the region $(0.5<r / B<1.5)$ so that far-field effects as well as the near-tip effects could be minimized. The displacement field data was analyzed using an over-deterministic least-squares analysis $[5,8]$.

Ideally, stress intensity factors can be evaluated using any of the two measured displacement components $u_{x}$ and $u_{y}$, or using transformed components in polar coordinates, namely radial $\left(u_{r}\right)$ and tangential $\left(u_{\theta}\right)$ displacements. Previous works by the authors $[\mathrm{xx}]$ and other investigators have shown that radial displacements offer a relatively robust estimation of crack tip stress intensity factors. Accordingly stress intensity factors presented were obtained by analyzing the radial displacement field. The details are avoided here for brevity.

In Fig. 3(a), mode-I stress intensity factor $\left(K_{\mathrm{I}}\right)$ histories for different fiber orientations are presented. In general, regardless of angles, mode-I stress intensity factors monotonically increase before crack initiation and attains a peak value close to crack initiation time. A steady increase can be explained by increasing crack tip loading up to the initiation event. More specifically, the peak values in Fig. 3(a) are somewhat different from one another. The $K_{\mathrm{I}}$ values at crack initiation (close to the peak value of history) increase modestly from $0^{\circ}$ and $15^{\circ}$ and they increase relatively rapidly for higher angles. For lower $\beta\left(v i z, 0^{\circ}\right.$ and $15^{\circ}$ ), due to lower resistance against crack initiation along the fiber direction, crack initiation occurs earlier. On the other hand, for higher $\beta\left(v i z ., 30^{\circ}\right.$ and $\left.45^{\circ}\right)$, crack initiation occurs much later after impact. Crack initiation times for the four cases considered are $33,54,63,84 \mu$ s for $\beta=0,15,30$ and $45^{\circ}$, respectively. The magnitude of stress intensity factor at crack initiation for different fiber orientation angles increases with increasing fiber orientation angle. 

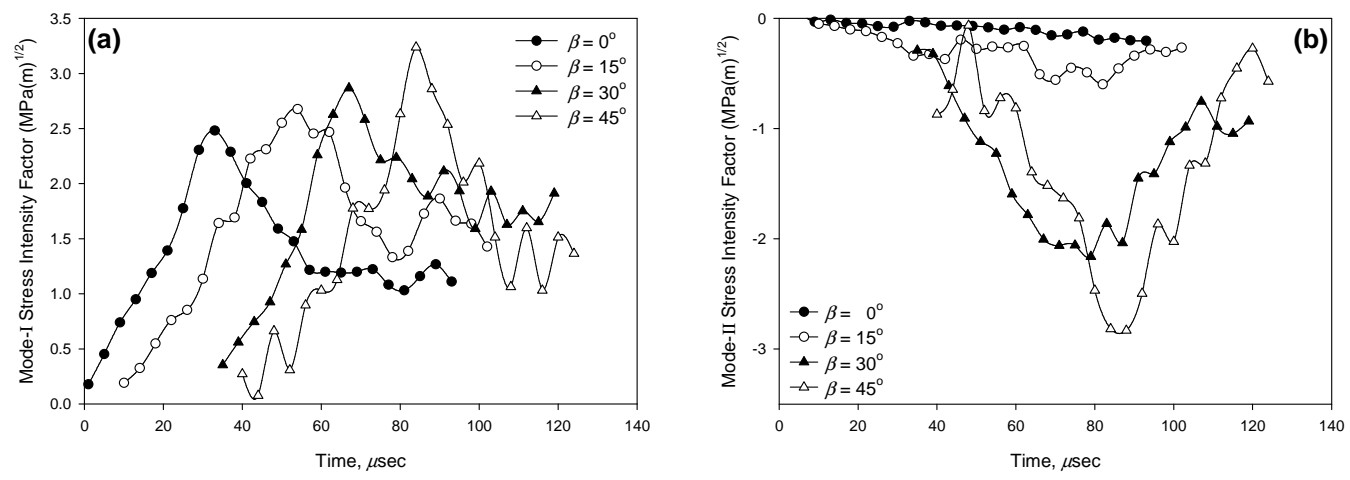

Figure 3. Mode-I stress intensity factor (a) and Mode-II stress intensity factor (b) against time for various angles under dynamic loading condition.

In Fig. 3(b), mode-II stress intensity factor $\left(K_{\mathrm{II}}\right)$ histories for the four fiber orientations are presented. Interestingly, in each case, mode-II stress intensity factors continue to remain negative as the crack propagates. This is unlike mixedmode fracture of isotropic brittle materials (for example, see Ref. [4]) where $K_{\text {II }}$ jumps from a negative to a near zero value as the crack initiates and remains same during crack growth in a direction normal to the local maximum tangential stress (or, the so-called $K_{\mathrm{II}}=0$ criterion).

Lastly, it is also instructive to compare stress intensity factors at initiation for all the four cases of $\beta$ examined. As expected, stress intensity factor at initiation increases with increasing fiber orientation angle: namely, 2.36, 2.54, 3.15, and $3.97 \mathrm{MPa}(\mathrm{m})^{1 / 2}$ for $0^{\circ}, 15^{\circ}, 30^{\circ}$, and $45^{\circ}$, respectively. The exponential relationship between fiber orientation angle and stress intensity factor at initiation becomes clearer, if stress intensity factor is expressed in a function of $E_{x x} / E_{y y}$ ratio, also known as the degree-of-anisotropy. Each fiber orientation angle can be uniquely expressed in terms of the degree-of-anisotropy. In the present work, the values are $20.8,7.5,2.4$, and 1.0 for $0^{\circ}, 15^{\circ}, 30^{\circ}$, and $45^{\circ}$, respectively. Upon plotting the effective stress intensity factor $\left(K_{e f f}=\sqrt{K_{I}^{2}+K_{I I}^{2}}\right)$ as a function of degree-of-anisotropy, an exponentially decay can be observed as shown in Fig. 4. Parhizgar et al. [10] reported a similar result for quasi-static fracture of glass fiber/epoxy system. Rowlands [11] also reported increasing critical stress intensity factor with increasing angle, but the trend was not an exponential variation possibly due to the multidirectional composite laminates he has studied. 


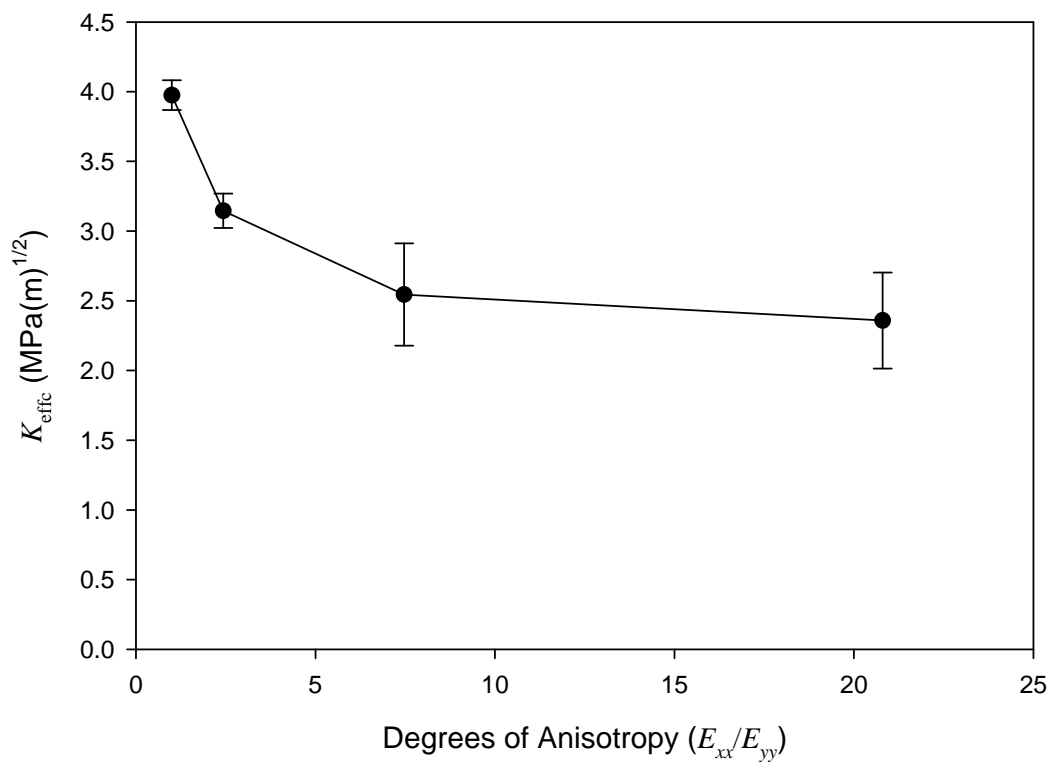

Figure 4. Effective stress intensity factor as a function of degree-of-anisotropy $\left(E_{x x} / E_{y y}\right)$. Error bars indicate standard deviation from three different values (one before and after crack initiation) due to uncertainty of crack initiation time.

\section{Concluding Remarks}

The DIC technique coupled with high-speed photography is shown to be effective for characterizing stress-wave induced fracture of unidirectional composite laminates. High-speed photography using a rotating mirror-type digital camera enables quantification of crack growth behavior. The optical method serves to determine full-field deformations in the vicinity of a dynamically growing crack tip and fracture parameters. An over-deterministic least-squares analysis of radial displacement components provide stress intensity factors.

The graphite epoxy laminates show differences in fracture responses depending upon the fiber orientation relative to the initial crack and loading directions. The maximum observed crack speed is the highest for mode-I conditions and decrease with fiber orientation angle $\beta$. With increasing $\beta$, crack takes longer to attain the maximum speed following initiation. Further, crack growth occurs under non-zero $K_{\text {II }}$ conditions. The effective stress intensity factor at crack initiation increases with increasing value of $\beta$. Alternatively, it is shown to decrease with the degreeof-anisotropy. Changes in crack tip shear deformations with $\beta$ are attributed to these differences. 


\section{Acknowledgments}

This research was sponsored financially by the NASA Langley Research Center through a grant to Auburn University, under Cooperative Agreement \#NNX07AC64A. Authors gratefully acknowledge the help of Dr. Phillip Bogert at NASA Langley Research Center for many helpful discussions and supplying the materials.

\section{References}

[1] C.A. Walker, Jamasri, Mixed-Mode Stress Intensity Factors in Finite, EdgeCracked Orthotropic Plates, J Strain Anal Eng Des 30 (2) (1995) 83-90

[2] D. Coker, A.J. Rosakis, Experimental Observations of Intersonic Crack Growth in Asymmetrically Loaded Unidirectional Composite Plates, Philos. Mag. A Phys. Condens. Matter Struct. Defects Mech. Prop. 81 (3) (2001) 571595

[3] F. Ansari, Experimental Analysis of Fracture in High Strength Cementitious Composites," in: J.F. Young (Eds.), Mat Res Soc Symp Proc, Very High Strength Cement-Based Materials Symposium, Materials Research Society, 42, 1985, pp. 101-110

[4] M.S. Kirugulige, H.V. Tippur, T.S. Denney, Measurement of Transient Deformations Using Digital Image Correlation Method and High-Speed Photography, Appl. Opt. 46 (24) (2007) 5083-5096

[5] M.S. Kirugulige, H.V. Tippur, Measurement of Surface Deformations and Fracture Parameters for a Mixed-Mode Crack Driven by Stress Waves Using Image Correlation Technique and High-Speed Photography, Strain (2008) accepted

[6] G.C. Sih, P.C. Paris, G.R. Irwin, On Cracks in Rectilinearly Anisotropic Bodies, Int. J. Fract. Mech. 1 (3) (1965) 189-203

[7] D.J. Chen, F.P. Chiang, Y.S. Tan, H.S. Don, Digital Speckle-Displacement Measurement Using a Complex Spectrum Method, Appl. Opt. 32 (11) (1993) 1939-1849

[8] Bruck, H.A., McNeill, S.R., Sutton, M.A., and Peters, W.H., "Digital Image Correlation Using Newton-Raphson Method of Partial Differential Correction," Exp Mech 29 (3) (1989) 261-268

[9] R.J. Sanford, Application of the Least-Squares Method to Photoelastic Analysis, Exp Mech 20 (6) (1980) 192-197

[10] S. Yoneyama, Y. Morimoto, M. Takashi, Automatic Evaluation of MixedMode Stress Intensity Factors Utilizing Digital Image Correlation, Strain 42 (1) (2006) 21-29

[11] S. Parhizgar, L.W. Zachary, C.T. Sun, Determination of Fracture Toughness of Unidirectionally Fiber-Reinforced Composites, in: G.C. Sih, 
V.P. Tamuzs (Eds.) Fracture of Composite Materials, Proceedings of the 2nd USA-USSR Symposium, Martinus Nijhoff, Hague, Boston, 1982, pp. 215-230

[12] R.E. Rowlands, Fracture of Anisotropic and Composite Materials, AMD Symposia Series (American Society of Mechanical Engineers, Applied Mechanics Division) 74 (1985) 15-31

[13] J. Lambros, A.J. Rosakis, Dynamic Crack Initiation and Growth in Thick Unidirectional Graphite/Epoxy Plates, Compos Sci Technol 57 (1) (1997) 5565

[14] C. Liu, Y. Huang, M.L. Lovato, M.G. Stout, Measurement of the Fracture Toughness of a Fiber-Reinforced Composite Using the Brazilian Disk Geometry, Int. J. Fract. 87 (3) (1997) 241-263

[15] S.K. Khanna, A. Shukla, Development of Stress Field Equations and Determination of Stress Intensity Factor during Dynamic Fracture of Orthotropic Composite Materials, Eng Fract Mech 47 (3) (1994) 345-359 